\title{
Analyse expérimentale de l'influence de l'âge à la première mise bas et du type génétique sur la productivité de la truie
}

\author{
C Legault ${ }^{1}, \mathrm{MC}$ Gauthier ${ }^{1 *}$, JC Caritez ${ }^{2}, \mathrm{H}$ Lagant 1 \\ 1 Station de génétique quantitative et appliquée, Centre de recherche Inra de Jouy-en-Josas, \\ 78352 Jouy-en-Josas; \\ 2 Domaine expérimental du Magneraud, Centre de recherche Inra de Poitou-Charentes, \\ 17700 Surgères, France
}

(Reçu le 31 mai 1994 ; accepté le 24 mars 1995)

Résumé - Le but de cette étude était de vérifier expérimentalement l'incidence de l'âge à la première mise bas sur le développement corporel, la longévité et la productivité des truies sur l'ensemble de leur carrière limitée à cinq cycles de reproduction. Pour cela, l'échantillon analysé est constitué de 61 truies F1 Landrace Français $\times$ Meishan (LF x MS) mises à la reproduction soit à l'âge de 5 mois (M5), soit à l'âge de 7 mois (M7) et 29 truies F1 Landrace $\times$ Large White (LF $\times L W)$ mises à la reproduction à l'âge de 7 mois (T7) et considérées comme témoins. Les truies à demi chinoises saillies à 5 mois ont la meilleure longévité et ont réalisé les meilleures performances de reproduction : le retard de développement corporel enregistré au cours du premier cycle se résorbe à partir de la deuxième mise bas. La productivité numérique par année de présence (de lâge de 150 jours au sevrage de la cinquième portée) est respectivement de 29,4 pour les $M 5,27,0$ pour les $M 7$ et 23,3 pour les T7. Cela se traduit par une économie de $27 \mathrm{~F}$ chez les M5 et de $19 \mathrm{~F}$ chez les M7 sur le prix de revient du porcelet par rapport aux T7. Néanmoins, ce progrès ne comble que partiellement la perte observée à la vente du porc charcutier terminal un quart chinois. Enfin, pour des truies conventionnelles $L W \times L F$, un avantage économique substantiel pourrait être obtenu rapidement en avançant d'un mois la mise à la reproduction.

porc / reproduction / âge première mise bas / type génétique / développement corporel / longévité I productivité numérique

Summary - Experimental study of the effects of age at first farrowing and dam genetic type on lifetime sow productivity. This experiment was designed to estimate the effects of age at first farrowing and dam genetic type on body development, longevity and productivity of sows on the basis of five successive farrowings. Sixty-one F1 French Landrace $x$ Meishan crossbred gilts ( $L F \times M S$ ) had a first mating either at 5 (M5) or at 7 months of age (M7) and were compared to 29 F1 French Landrace $x$ Large

*Adresse actuelle : Chambre d'agriculture des Deux-Sèvres, 79000 Niort, France 
White crossbred gilts ( $L F \times L W)$ mated at 7 months of age (T7) and considered as controls. The highest longevity as well as the best overall reproductive performances were observed in $L F \times M S$ sows mated at 5 months. The delay in body development observed over the first reproductive cycle became nonsignificant after the second or the third reproductive cycle. The numerical productivity estimated from the age of 150 days to weaning of the fifth litter was 29.4, 27.0 and 23.3 pigs weaned/sow/year for M5, $M 7$ and $T 7$ sows, respectively. As a consequence, the cost of the weaned pig was reduced by $27 \mathrm{~F}$ in $M 5$ and by $19 \mathrm{~F}$ in $M 7$ as compared to $T 7$ pigs. However, under intensive management systems, this benefit is not large enough to reduce the loss due to lower growth and carcass performance in 1/4 Chinese terminal slaughter pigs.

\section{pig / reproduction / age at first mating / dam genetic type / body development / longevity / numerical productivity}

\section{INTRODUCTION}

Face à des coûts de production croissants, l'éleveur se trouve dans l'obligation d'intensifier l'utilisation de ses truies reproductrices. La réduction des périodes improductives telles que celle allant de la fin de l'engraissement à la première mise bas peut être un facteur de progrès en diminuant le prix de revient du porcelet. En France, il s'avère que l'âge à la première mise bas, qui est de l'ordre de 360 jours, n'a pratiquement pas varié depuis 1979 (Gauthier et al, 1992) Pourtant, de nombreuses études aboutissent à la conclusion que tout retard à la première mise bas a une incidence économique significative toujours défavorable (Legault et Dagorn, 1973 ; Chapman et al, 1978 ; Brook et Smith, 1980 ; Baker et Morris, 1982 ; Noguéra et Guéblez, 1984 ; Vermeer et Slijkkuis, 1989 ; Young et al, 1990 ; Lynch et al, 1994). Selon les modèles économiques proposés par Tess et al (1983) pour les États-Unis et par Bidanel (1989) pour la France, les variations de l'âge à la première mise bas expliquent 3 à $4 \%$ de celles de la rentabilité d'un élevage du type "naisseur-engraisseur'. Enfin, Schukken et al (1994) ont estimé que l'optimum économique pour la mise à la reproduction des cochettes croisées Large White $\times$ Landrace se situe entre les âges de 200 et 220 jours.

Plusieurs raisons sont avancées pour expliquer la stabilité de la situation chez les éleveurs. Nous mentionnerons en premier lieu la barrière biologique représentée par le déclenchement de la puberté (vers l'âge de 6-7 mois dans les races européennes). Par ailleurs, de nombreux professionnels pensent qu'une mise à la reproduction trop précoce pourrait avoir des effets défavorables sur la production, le développement corporel et la longévité de la truie. Enfin le transfert des cochettes de remplacement de leur élevage d'origine (multiplicateurs) vers leur élevage d'utilisation (naisseurs) est une opération relativement longue dont la planification routinière est difficile à remettre en question.

Nous nous proposons d'étudier les conséquences de l'âge à la première mise bas sur un matériel expérimental représenté par des cochettes F1 Landrace $\times$ Meishan mises à la reproduction soit vers l'âge de 5 mois (M5), soit à un peu plus de 7 mois (M7) en comparaison à des truies témoins Landrace $\mathrm{x}$ Large White mises également à la reproduction à 7 mois (T7). Toutes les études citées en référence ont été réalisées sur des animaux conventionnels (essentiellement Large White $x$ Landrace), chez lesquels l'âge moyen à la première fécondation est toujours supérieur à 200 jours. Le recours à des cochettes demi-chinoises s'explique par leur précocité sexuelle exceptionnelle (Legault et Caritez, 1983) qui permet d'envisager une réduction de l'âge à la première mise bas de 2 à 3 mois. Les résultats préliminaires de cette étude ont fait l'objet d'une première publication (Gauthier et al, 1992). 


\section{MATÉRIEL ET MÉTHODES}

\section{Animaux et dispositif expérimental}

Seize truies Meishan (MS) et 15 truies Large White (LW) du troupeau expérimental du Magneraud (17700 Surgères) ont été inséminées avec de la semence de 15 verrats Landrace Français (LF) en service dans les centres d'insémination artificielle (sur les 15 verrats LF utilisés, 12 l'ont été sur les deux lots de truies). Les produits femelles de ces croisements représentent le matériel animal de base de l'expérience. Le type génétique LF x LW servira de témoin. À partir de ces produits $\mathrm{F} 1$ et à raison d'un choix de deux porcelets femelles par portée, trois lots de cochettes ont été constitués. L'échantillonnage des cochettes destinées à représenter les deux lots M5 et M7 a été fait intraportée de manière à éviter toute différence significative pour les critères de croissance et d'adiposité : $89,5 \mathrm{~kg}$ chez les M5 contre $87,8 \mathrm{~kg}$ chez les M7 pour le poids à 154 jours et $24,3 \mathrm{~mm}$ chez les M5 contre $24,1 \mathrm{~mm}$ chez les M7 pour l'épaisseur du lard dorsal au même âge. Les valeurs correspondantes chez les cochettes $T 7$ étaient respectivement de $96,4 \mathrm{~kg}$ et $19,1 \mathrm{~mm}$. Ces cochettes ont été inséminées pour $81 \%$ avec de la semence de verrats Piétrain ; le changement de "verrat terminal» qui est intervenu en fin d'expérience a un effet qui se trouve confondu avec celui des rangs de portée 4 et 5 .

Un ensemble de 90 cochettes ont été intégrées dans les bandes du troupeau par lots de contemporaines (intervalle entre bandes de 3 semaines). Un seul retour en chaleur après l'œstrus post-sevrage a été toléré et les truies ont poursuivi leur carrière jusqu'à la $5^{\mathrm{e}}$ portée au terme de laquelle elles ont été réformées dès le sevrage. Le sevrage a été pratiqué à $29,2 \pm$ 2,3 jours.

\section{Contrôles effectués}

Toutes les truies ont été pesées à la première saillie, puis à l'entrée en maternité et le jour du sevrage pour chacun des cinq cycles de reproduction. L'épaisseur de lard dorsal a été mesurée à l'aide d'un appareil à ultra-sons pour l'ensemble des truies (au niveau de la dernière côte à $10 \mathrm{~cm}$ de part et d'autre de la colonne vertébrale) à l'entrée en maternité et au sevrage pour chacun des cycles.

L'ensemble des critères de reproduction a été soumis au contrôle habituel : dénombrement des porcelets nés totaux, nés vivants, sevrés et pesées individuelles des porcelets à la naissance et à 21 jours d'âge. Enfin, la consommation d'aliment de la truie en maternité a été mesurée individuellement et ajustée à la durée constante de 30 jours par extrapolation de la consommation au cours des 2 derniers jours d'allaitement.

\section{Analyse statistique}

Les données recueillies sur les 362 portées dans le cadre de l'expérience ont été analysées selon la procédure Blup-Modèle Animal en utilisant le logiciel Pest mis au point par Groeneveld et al (1990). Le modèle d'analyse prend en compte 3 effets fixés : le traitement (trois niveaux : M5, M7 et $\mathrm{T7}$ ), le rang de portée (cinq niveaux), la saison correspondant à quatre bandes de mise bas consécutives et les interactions de 1 er ordre entre les trois facteurs. Deux effets aléatoires ont été considérés : la truie et un effet correspondant à l'environnement permanent propre à chaque portée. Enfin, les comparaisons de fréquences ont été réalisées par la méthode du $\chi^{2}$.

\section{Productivité et base économique}

Toutes ces données nous permettent d'estimer la productivité numérique des trois lots de truies. L'estimation habituelle de ce paramètre sur la base de l'année de vie reproductive ( $P n$ : première à la dernière mise bas) ne permet pas d'effectuer une analyse économique précise. C'est pourquoi nous préférons l'estimation de cette productivité sur la base de l'année de présence dans le troupeau $\left(P^{\prime} n\right)$. Dans le cadre de notre étude, le temps de présence a été fixé arbitrairement de l'âge de 150 jours au sevrage de la cinquième portée.

La productivité $P$ 'n est estimée conformément à la formule suivante :

$$
P^{\prime} n=\frac{T p s \times N \times 365}{i 1+(N-1) \times M B+L}
$$


où $T p s=$ taille de la portée au sevrage ; $N=$ nombre de portées sevrées par truie ; $i 1=$ intervalle entre l'âge de 150 jours (fin de l'engraissement) et la première mise bas ; $M B=$ intervalle entre mise bas ; $L=$ durée d'allaitement ( 28 jours).

Le prix de revient du porcelet au sevrage a été estimé en tenant compte des temps de présence réels des truies dans le troupeau et du nombre total de porcelets sevrés au cours de la même période pour chacun des trois traitements. Par conséquent, la période de référence va de l'âge de 150 jours au sevrage de la dernière portée. Le prix de revient du porcelet $(P r)$ correspond donc à la formule suivante :

$$
P r=\frac{C T}{S}+C 1
$$

où $C T=$ coût d'entretien total de la truie ; $S=$ nombre total de porcelets sevrés par truie au cours de la période de référence ; $c 1=$ coût alimentaire du porcelet «sous la mère».

Le coût total d'entretien de la truie se décompose en un coût alimentaire et en frais de personnel, amortissement, frais généraux, etc. L'alimentation de la truie représente $45 \%$ des charges totales selon le schéma naisseur-vente au sevrage (ITP, 1992). Le coût alimentaire de la truie au cours d'un cycle de reproduction a été calculé sur les bases suivantes (le coût par cycle étant multiplié par le nombre de portées réellement sevrées) : $2,7 \mathrm{~kg} / \mathrm{jour}$ en jeune reproducteur (de l'âge de 150 jours à la première saillie) ; 84 jours à $2,7 \mathrm{~kg} / \mathrm{jour}$ (début de gestation); 30 jours à $3,2 \mathrm{~kg} / \mathrm{jour}$ (fin de gestation); 30 jours à 5,2 $\mathrm{kg} / \mathrm{jour}$ (lactation); 6 jours à 2,7 kg/jour (du sevrage à la fécondation).
La teneur moyenne de l'aliment "gestation" (ou «jeunes reproducteurs") et de l'aliment «lactation" est respectivement de 15 et $16 \%$ pour le taux de matière azotée totale et de 3075 et $3200 \mathrm{kcal}$ d'énergie digestible $/ \mathrm{kg}$ de matière sèche. Le prix de cet aliment est de 1,20 F/kg (valeur moyenne pour l'élevage en 1993). Nous considérons que le porcelet consomme $0,8 \mathrm{~kg}$ d'aliment en cours d'allaitement (gaspillage compris) à $6,10 \mathrm{~F} / \mathrm{kg}$.

\section{RÉSULTATS}

\section{Carrière des truies}

Sur 90 cochettes mises à la reproduction, 84 (soit $93,3 \%$ ) ont mis bas au moins une fois. Les échecs de mise à la reproduction ont affecté 10,3\% des cochettes du lot T7 (deux femelles pour absence d'œstrus et un pour non fécondation après la saillie). Chez les truies F1 LF x MS, 4,9\% des cochettes ont subi cet échec (un pour absence de chaleur et deux pour un $2^{\mathrm{e}}$ retour en chaleur après saillie). Cependant, les fréquences de ces échecs ne diffèrent pas significativement selon le type génétique $\left(\chi^{2} 1 d d l=\right.$ 0,99).

La figure 1, établie à partir des femelles ayant mis bas au moins une fois, illustre la «fonte» du troupeau selon le traitement.

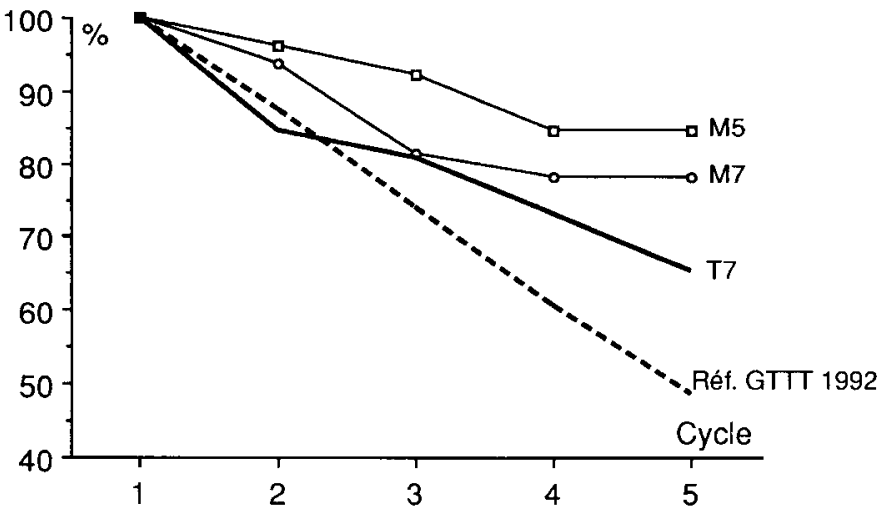

Fig 1. Courbe représentative de la fonte du troupeau selon le type génétique et l'âge à la première mise bas en référence à la moyenne nationale (GTTT, 1992). 
Nous pouvons observer que la meilleure longévité se rencontre chez les femelles M5 (84,6\% ayant sevré leur cinquième portée), celles-ci sont suivies par les M7 $(78,1 \%)$ puis par les T7 $(65,4 \%)$. Quel que soit le type de comparaison (M5 et M7 regroupés ou séparés), les fréquences des truies ayant sevré leur cinquième portée ne diffèrent pas significativement selon le traitement (valeurs de $\chi^{2}$ non significatives au seuil de $5 \%$ ). Cependant la fonte observée dans notre troupeau expérimental est significativement inférieure à celle estimée dans le troupeau national français par le programme national de gestion technique des troupeaux de truies : $46,3 \%$ des truies ont atteint le cinquième sevrage en 1992.

Les causes de réforme sont répertoriées dans le tableau I. Les troubles de locomotion $(31,6 \%)$ et les échecs de reproduction $(42,1 \%)$ sont les principales causes de réforme, les faibles effectifs ne permettant pas d'aboutir à des conclusions sur le rôle du type génétique ou du traitement.

\section{Développement corporel des truies}

Les résultats de l'analyse statistique des variations pondérales des truies sont illustrés par la figure 2. À la première saillie, conformément au protocole, les cochettes M5 ont 66 jours de moins que les cochettes $M 7$ et T7. Leur poids à ce stade est inférieur de $52 \mathrm{~kg}$ à celui des $M 7$ et de $56 \mathrm{~kg}$ à celui des T7. Dès la première mise bas, ces différences de poids s'atténuent puisqu'elles ne sont plus respectivement que de 36 et $44 \mathrm{~kg}$. La réduction de cet écart pondéral se poursuit et les différences cessent d'être significatives dès le quatrième cycle à la mise bas. Cependant, le poids au sevrage chez les M5 est toujours significativement inférieur à celui des $M 7$ en raison de pertes de poids plus élevées en cours de lactation. Quant aux différences pondérales entre $M 7$ et T7, elles ne sont pas significatives.

Les résultats de l'analyse des gains de poids en cours de gestation et des pertes de poids en cours de lactation sont rassemblés dans le tableau II. Les gains de poids en cours de gestation sont toujours supérieurs chez les truies M5 ; cette différence est significative à l'un ou l'autre des traitements aux cycles 1, 3 et 4 . Les pertes de poids en cours de première lactation sont significativement plus faibles chez les M5 (34 kg contre $45 \mathrm{~kg}$ ). À partir du deuxième cycle, l'effet du traitement sur ces variations (45 à $62 \mathrm{~kg}$ ) n'est plus significatif.

Tableau I. Répartition des causes de réforme (avant la cinquième portée).

Causes de réforme

Échecs de reproduction
Troubles de locomotion
Infections urogénitales
Divers

Ensemble
Traitement

N \%

$\begin{array}{lr}2 & 50,0 \\ 2 & 50,0 \\ 0 & 0,0 \\ 0 & 0,0\end{array}$

4100,0
$M 7$

\begin{tabular}{cr}
\multicolumn{2}{c}{$M 7$} \\
$N$ & $\%$ \\
& \\
& \\
4 & 57,1 \\
1 & 14,3 \\
2 & 28,6 \\
0 & 0,0
\end{tabular}

$7 \quad 100,0$
Ensemble

\section{T7}

$N$

$\%$

N $\%$

$\begin{array}{rrrr}2 & 25,0 & 8 & 42,1 \\ 3 & 37,5 & 6 & 31,6 \\ 1 & 12,5 & 3 & 15,8 \\ 2 & 25,0 & 2 & 10,5 \\ 8 & 100,0 & 19 & 100,0\end{array}$




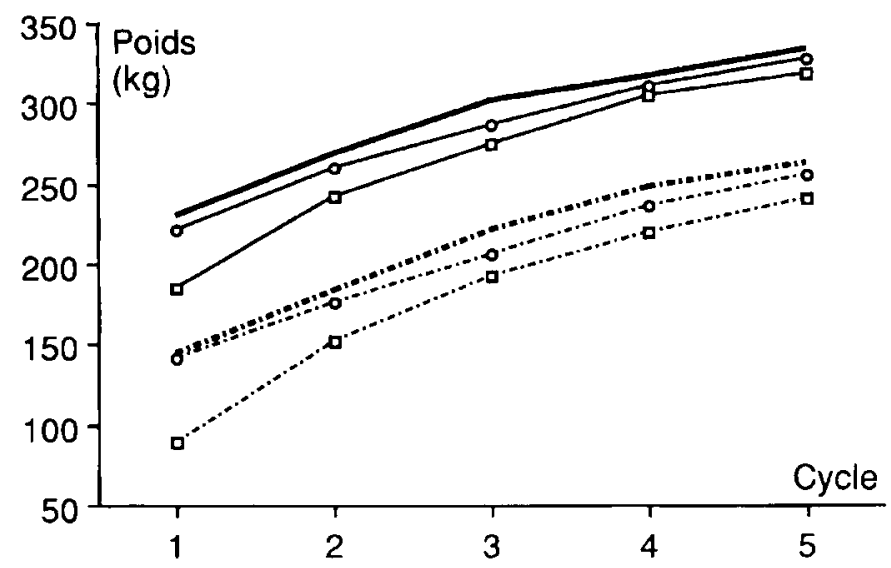

Fig 2. Courbes d'évolution pondérale à la saillie et à la mise bas en fonction du traitement. - - - - M5, poids à la saillie ; $-\mathrm{J}-\mathrm{M} 5$, poids à la mise bas ; - -O- $M 7$, poids à la saillie; - M7, poids à la mise bas ; $---T 7$, poids à la saillie ; - $\mathrm{T} 7$, poids à la mise bas.

La figure 3 retrace les variations de l'épaisM7 et T7 est de $6,9 \mathrm{~mm}$, bien que ces animaux aient le même âge. Par la suite, les seur de lard dorsal de ces truies. Les femelles à demi chinoises ont une carcasse naturellement plus grasse que celle des témoins. Ainsi, à la première saillie, la différence entre témoins demeurent significativement moins gras que les truies LF x MS (écart moyen de 4 à $6 \mathrm{~mm}$, à la mise bas comme au sevrage).

Tableau II. Variations de poids (en $\mathrm{kg}$ ) moyennes au cours de la gestation et la lactation selon le cycle de reproduction.

Premier cycle

Gain en gestation

Pertes en lactation

Deuxième cycle

Gain en gestation

Pertes en lactation

Troisième cycle

Gain en gestation

Pertes en lactation

Quatrième cycle

Gain en gestation

Pertes en lactation

Cinquième cycle

Gain en gestation

Pertes en lactation $93,2^{\mathrm{a}}$

$33,5^{\mathrm{a}}$

$94,3^{\mathrm{a}}$

$49,4^{\mathrm{a}}$

$87,3^{a}$

$54,8^{a}$

$85,9^{\mathrm{a}}$

$62,4^{\mathrm{a}}$

$78,0^{a}$

$52,1^{\text {a }}$ $83,8^{b}$

$44,7^{b}$

$88,4^{\text {a }}$

$51,4^{\mathrm{a}}$

$90,6^{\mathrm{a}}$

$45,1^{\text {a }}$

$77,9^{b}$

$49,0^{a}$

$81,2^{\mathrm{ab}}$

$53,6^{a}$

$78,2^{\mathrm{ab}}$

$70,3^{b}$

$53,8^{\mathrm{a}}$

$72,6^{a}$

$45,7^{a}$

Les valeurs pour un cycle et une période donnée portant la même lettre ne diffèrent pas significativement au seuil de $5 \%$. 
Fig 3. Courbes des variations d'adiposité au cours des cycles de reproduction selon le traitement. $-\square-\mathrm{M} 5$; - O-M7 ; T7 ; SA : saillie ; $M B$ : mise bas ; SEV : sevrage.

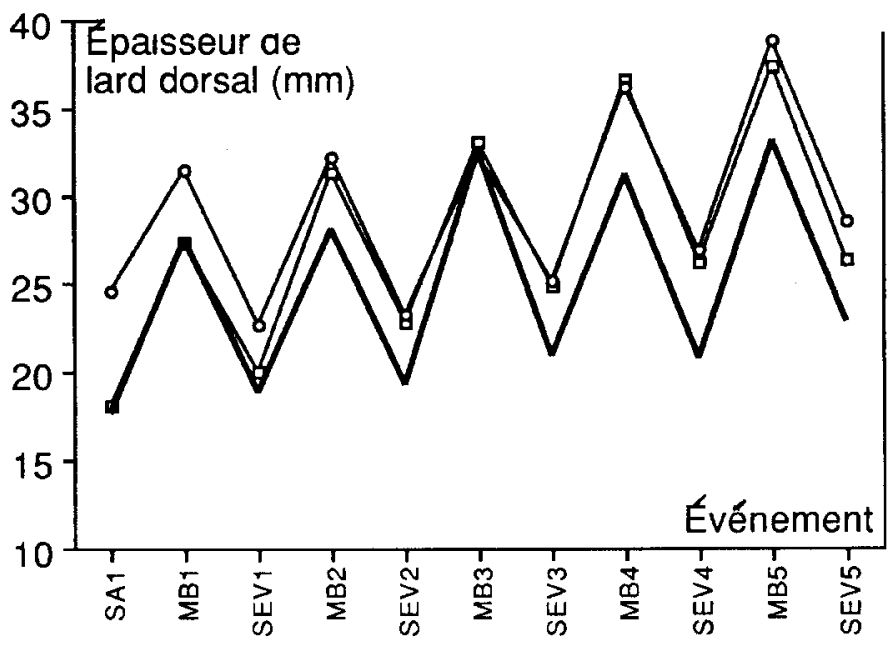

II semble intéressant de comparer les pertes d'adiposité en cours de lactation selon le cycle de reproduction et le traitement. L'analyse statistique montre qu'au cours de la première lactation les truies M5 perdent significativement moins de gras que les autres $(7,3$ contre $8,8 \mathrm{~mm})$. Pour les cycles suivants, les pertes d'adiposité sont dans leur ensemble indépendantes du traitement (entre 8,6 et $11,7 \mathrm{~mm}$ ).

\section{Performances de reproduction}

Le tableau III rassemble les valeurs estimées des composantes de la reproduction pour chacun des trois traitements. Nous ne nous attarderons pas sur l'effet bien connu du rang de portée qui est hautement significatif pour l'ensemble des variables, exception faite du taux de survie (fig 4). Comme on pouvait s'y attendre, la prolificité des truies à demi chinoises est significativement supérieure à celle des témoins.

La comparaison entre les deux lots de truies à demi chinoises ne fait apparaître aucune différence significative pour leur prolificité sauf en première portée où les truies M7 expriment un avantage significatif pour ce critère (cf fig 4). Cependant, aucune différence significative entre M5 et M7 n'est observée pour le poids de la portée à la naissance et à 21 jours quel que soit le numéro du cycle. Ainsi, à titre indicatif, pour le poids de la portée à 21 jours (estimateur de la production laitière), les valeurs estimées sont de $72 \mathrm{~kg}$ pour les M5 et les M7 et $65 \mathrm{~kg}$ pour les T7. En première portée, ces valeurs étaient respectivement de 60,65 et $56 \mathrm{~kg}$.

Le contrôle de l'alimentation des truies au cours de la lactation ne fait apparaître aucune différence significative entre les lots (156 à $160 \mathrm{~kg}$ d'aliment concentré consommé en moyenne).

Comme l'indique le tableau III, nous noterons enfin l'absence de toute différence significative pour le rythme de reproduction (intervalle entre mise bas et intervalle sevrage-fécondation).

Le nombre de portées $(N)$ réellement sevrées par les truies de chacun des trois lots est de 4,6 pour les M5, de 4,3 pour les M7 et de 4,0 pour les T7. Ces valeurs sont tout à fait comparables à celles estimées dans le troupeau français par le programme national GTT $(4,6$ portées sevrées par truie réformée en 1992 et 1993). La durée 
moyenne de gestation est de 114 jours pour chacun des trois lots.

Le nombre de porcelets sevrés par truie par année de vie reproductive $(P n)$ est respectivement de $30,4,29,6$ et 25,9 chez les M5, M7 et les T7. Le nombre de porcelets sevrés par année de présence ( $P$ ' $n$ ) est respectivement de $30,3,26,8$ et 23,3 chez les M5, M7 et T7.

\section{Bilan économique}

Les prix de revient des porcelets au sevrage, comme l'indique le tableau IV, sont res- pectivement de 112,124 et $141 \mathrm{~F}$ pour les mères M5, M7 et T7. L'économie réalisée par une mise à la reproduction plus précoce est de $12 \mathrm{~F}$ par rapport aux M7. Pour des truies saillies au même âge, les truies à demi chinoises plus prolifiques permettent de réduire le prix de revient du porcelet de $18 \mathrm{~F}$ par rapport aux témoins.

\section{DISCUSSION ET CONCLUSION}

Le premier enseignement de cette étude est d'effacer les craintes que l'on pouvait avoir sur les conséquences néfastes d'une

Tableau III. Valeurs moyennes des composantes de la reproduction selon le traitement.

\begin{tabular}{|c|c|c|c|}
\hline & M5 & $M 7$ & $T 7$ \\
\hline Nombre de nés totaux & $14,1^{\mathrm{a}}$ & $14,0^{\mathrm{a}}$ & $12,4^{b}$ \\
\hline Nombre de nés vivants & $13,7^{a}$ & $13,6^{a}$ & $11,9^{\mathrm{b}}$ \\
\hline Nombre de sevrés & $12,4^{a}$ & $12,1^{\mathrm{a}}$ & $10,6^{\mathrm{b}}$ \\
\hline Taux de survie (\%) & $89,9^{a}$ & $87,7^{\mathrm{a}}$ & $89,4^{a}$ \\
\hline Poids portée naissance $(\mathrm{kg})$ & $18,3^{a}$ & $17,6^{\mathrm{a}}$ & $17,8^{\mathrm{a}}$ \\
\hline Poids portée 21 jours $(\mathrm{kg})$ & $71,9^{a}$ & $71,8^{a}$ & $64,9 \mathrm{a}$ \\
\hline Intervalle entre mise bas (jour) & $149,1^{\mathrm{a}}$ & $149,1^{a}$ & $149,2^{\mathrm{a}}$ \\
\hline Intervalle sevrage - saillie féc (jour) & $6,2^{\mathrm{a}}$ & $6,1^{\mathrm{a}}$ & $7,2^{\mathrm{a}}$ \\
\hline
\end{tabular}

Les valeurs portant la même lettre ne diffèrent pas significativement au seuil de $5 \%$.

Tableau IV. Paramètres de l'analyse économique selon le traitement.

$\begin{array}{lccr} & M 5 & M 7 & T 7 \\ \text { Nombre moyen de portées } & & & \\ P n & 4,6 & 4,3 & 4,0 \\ P^{\prime} n \text { a } & 30,4 & 29,6 & 25,9 \\ \text { Nombre de porcelets sevrés par truie } & 30,3 & 26,8 & 23,3 \\ \text { Coût total d'entretien de la truie (en F) } & 55,7 & 52,5 & 52,4 \\ \text { Prix de revient du porcelet au sevrage (en F) } & 6094 & 6173 & 5777 \\ & 112 & 124 & 141\end{array}$

\footnotetext{
a Le temps de présence va de l'âge de 150 jours au sevrage de la dernière portée.
} 

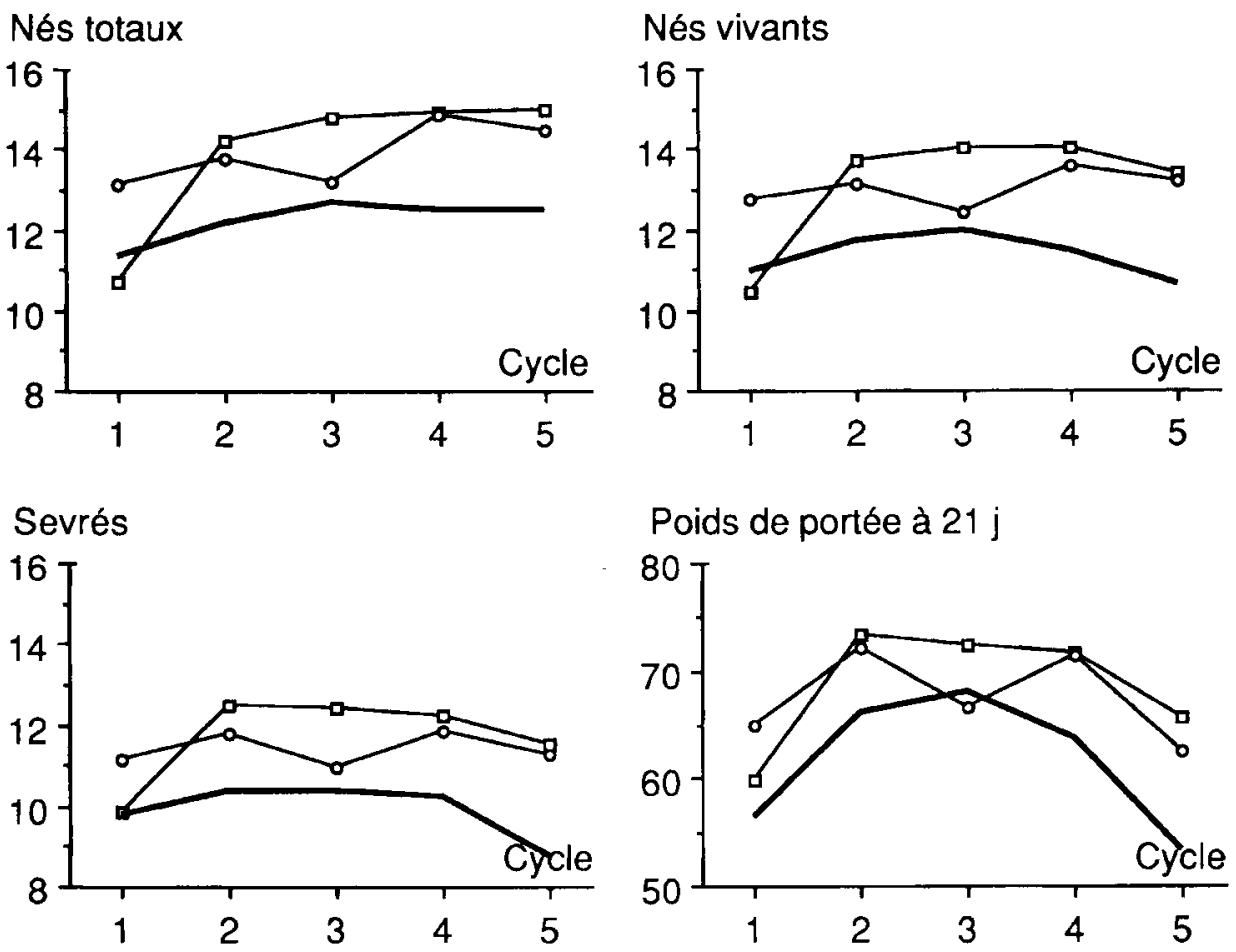

Fig 4. Évolution de la taille de portée à la naissance et au sevrage et du poids de la porté à 21 jours au cours des cinq cycles de reproduction selon le traitement. - ـ- M5; - $\mathrm{O}-\mathrm{M} 7 ;-\mathrm{T}$.

mise à la reproduction précoce des truies. En effet, les cochettes saillies dès l'âge de 5 mois sont celles qui ont effectué la meilleure carrière tant au niveau de la longévité que de la prolificité et de l'aptitude laitière (poids de la portée à 21 jours) : comme l'avaient constaté plusieurs auteurs (Brooks et Smith, 1980 ; Young et al, 1990 ; Lynch et al, 1994), les retards enregistrés au premier cycle pour le développement corporel et la prolificité s'estompent dès les deuxième ou troisième cycles de reproduction. En matière de longévité, ces résultats confirment les observations faites à très grande échelle chez les éleveurs par Noguera et Guéblez (1984) qui soulignaient l'existence d'une liaison négative entre l'âge à la première mise bas et le nombre de por- tées sevrées par truie réformée. D'autres études constatent l'indépendance entre la longévité et l'âge à la première mise bas (Young et al, 1990 ; Lynch et al, 1994).

En dépit d'une production laitière plus élevée, les truies demi-chinoises ne perdent pas plus de gras dorsal en cours de lactation que les témoins; ce fait suggère que ces animaux ont été trop bien alimentés et qu'ils ont moins puisé dans leurs réserves corporelles que les témoins. Une économie alimentaire aurait donc pu être réalisée sur ces types génétiques comme le suggéraient des résultats antérieurs (Legault et Caritez, 1983). En fait, la meilleure aptitude des truies demi-chinoises à mobiliser leurs réserves corporelles en cours de lactation est surtout appréciable dans un milieu diffi- 
cile marqué par d'importantes insuffisances nutritionnelles (Delate et al, 1991).

De nombreuses études évoquées dans l'introduction ont clairement démontré que tout retard à la mise à la reproduction chez la jeune truie se traduit par une augmentation du prix de revient des porcelets sevrés sur l'ensemble de leur carrière. En recourant à des femelles demi-chinoises dont la grande précocité sexuelle est connue (Legault et Caritez, 1983), la présente étude va plus loin en envisageant une mise à la reproduction dès l'âge de 5 mois, soit environ 90 jours plus tôt que la moyenne nationale (Gauthier et al, 1992). Toutefois, il nous reste à évaluer dans quelle mesure le gain réalisé sur le coût de production du porcelet permet de compenser la perte enregistrée sur la marge brute par porc charcutier un quart chinois. Cette perte n'a pu être estimée rigoureusement dans cette expérience. Néanmoins, sur la base de résultats antérieurs correspondant à des truies demi-chinoises saillies par des verrats terminaux spécialisés (Legault et al, 1985 ; Guéblez et al, 1987 ; Bidanel et al, 1989), le produit terminal un quart-chinois est accrédité d'environ quatre points de taux de muscle en moins par rapport aux témoins et de 0,07 point d'indice de consommation en plus. La comparaison des marges brutes prenant en compte la diminution du prix de vente des carcasses, le supplément de consommation d'aliment et les variations du prix de revient du porcelet se traduisent en définitive par une diminution de la marge brute de $38 \mathrm{~F}$ chez les M5 et de $50 \mathrm{~F}$ chez les M7 par rapport aux T7. Ces résultats démontrent une nouvelle fois qu'en élevage intensif conventionnel la meilleure façon de valoriser la haute prolificité des races chinoises n'est pas le croisement discontinu, mais leur intégration dans des lignées composites sinoeuropéennes comme l'ont suggéré les travaux théoriques de Bidanel (1989) et le confirment les premiers résultats de Naveau et al (1993). Or, dans une lignée composite, l'âge à la puberté se maintient théoriquement dès la deuxième génération à un niveau correspondant à la moyenne des races parentales diminuée de la moitié de l'effet d'hétérosis. Pour l'instant, cette prédiction théorique n'a pas encore été clairement vérifiée.

Constatant que l'âge à la première mise bas s'est stabilisé vers 360 jours dans le troupeau français (Gauthier et al, 1992), cette étude démontre qu'il est tout à fait possible d'avancer d'au moins 1 mois la première saillie chez les cochettes conventionnelles Large White $\times$ Landrace, sans compromettre ni leur productivité, ni leur longévité. Une proportion croissante d'éleveurs modernes a atteint le niveau de technicité requis pour faire face à cette nouvelle avancée qui réduirait de 5 à $6 \mathrm{~F}$ (soit d'environ $4 \%$ ) le prix de revient du porcelet au sevrage. En fait, si cette technique nouvelle est directement transposable chez les éleveurs assurant eux-mêmes le renouvellement de leurs cochettes, la difficulté tient au fait que la majorité des éleveurs s'approvisionne chez des multiplicateurs spécialisés, ce qui a pour effet de retarder très sensiblement la disponibilité de ces cochettes pour la mise à la reproduction (quarantaine, adaptation à un nouveau milieu, synchronisation, etc) ; c'est pourquoi l'extension de la pratique de la livraison des cochettes parentales dès l'âge de 4 semaines permettrait de surmonter cette difficulté. Rappelons enfin qu'une première saillie vers l'âge de 7 mois correspond aux conclusions de Shukken et al (1994) pour lesquels ce stade représente un optimum conciliant au mieux les paramètres biologiques de ce type génétique et l'économie de la production.

En conclusion, cette étude a d'abord démontré que les truies demi-chinoises étaient parfaitement adaptées à une mise à la reproduction dès l'âge de cinq mois sans que cela soit préjudiciable au déroulement de leur carrière. Cependant, dans 
les conditions d'un élevage intensif moderne, l'économie réalisée sur le coût de production du porcelet ne compense que partiellement la perte observée chez le produit terminal un quart chinois. Pour des truies conventionnelles Large White $x$ Landrace, un avantage économique substantiel pourrait être obtenu rapidement en avançant la mise à la reproduction d'un mois, recommandation à réserver aux élevages bénéficiant de la meilleure technicité.

\section{RÉFÉRENCES}

Baker RL, Morris CA (1982) Selection for early puberty and increased fertility at first mating. In : $2^{e}$ Congrès mondial de génétique appliquée à l'élevage, Madrid, 4-8 octobre, 1982, V, 282-293

Bidanel JP (1989) Étude de stratégies de valorisation en croisement de la race porcine Meishan. III. Evaluation comparée de différents systèmes de croisement. Journées Rech Porcine en France 21, 361. 366

Bidanel JP, Caritez JC, Fleury J, Gruand J, Legault C (1989) Étude de stratégies de valorisation en croisement de la race porcine Meishan. II. Estimation des paramètres de croisement pour les caractères de production. Journées Rech Porcine en France 21, 353-360

Brook PH, Smith DA (1980) The effect of mating age on the reproductive performance, food utilisation and liveweight change of the female pig. Livest Prod Sci $7,67-78$

Chapman JD, Thompson LH, Gaskins CT, Tribble LF (1978) Relationship of age at first farrowing and size of first litter to subsequent reproductive performance in sows. J Anim Sci 47, 780-787

Delate JJ, Le Guyadec P, Le Duot P, Duclos JM (1991) Croissance et reproduction des porcs rustiques d'origine trançaise selon le milieu d'élevage en Haïti. Journées Rech Porcine en France 23, 381-388

Gauthier MC, Legault C, Caritez JC (1992) Étude expérimentale de l'incidence de l'àge à la première mise bas sur la carrière des truies reproductrices. Joumées Rech Porcine en France 24, 337-344

Groeneveld E, Kovac M, Wang T (1990) PEST, a general purpose BLUP package for multivariate prediction and estimation. In : 4th World Congress in genetics applied to livestock production, Edinburgh, Scotland, 23-27 July, XIII, 488-491

Guéblez R, Bruel L, Legault C (1987) Évaluation des croisements sino-européens en condition de terrain en France : bilan général. Journées Rech Porcine en France 19, 25-32

ITP (1992) Le porc par les chiffres. ITP Éd, $40 p$

Legault C, Caritez JC (1983) L'expérimentation sur le porc chinois en France. I. Performances de reproduction en race pure et en croisement. Génét Sél Évol 15, 223-240

Legault C, Dagorn J (1973) Incidence de l'âge à la première mise bas sur la productivité de la truie. Journees Rech Porcine en France Paris, 5, 227-237

Legault C, Sellier P, Caritez JC, Dando P, Gruand J (1985) Expérimentation sur le porc chinois en France. II. Performances de production en croisement avec les races européennes. Génét Sél Evol 17, 133-152

Lynch PB, Hanrahan TJ, Arkins S (1994) Effect of age at first mating on lifetime performance of sows under two weaning regimes. Anim Prod 58, 470 (abstr)

Naveau J, Ducos A, Bidanel JP, Bazin C (1993) Résultats de huit générations de sélection sur la croissance et la composition corporelle de la lignée composite sino-européenne Tia Meslan. Journées Rech Porcine en France 25, 65-68

Noguera JL, Guéblez R (1984) Incidence de l'âge à la première mise bas et de la taille de la première portée sur la carrière de la truie. Journées Rech Porcine en France 16, 135-144

SAS (1992) SAS user's guide. Version 6. SAS Institut Inc, Cary, NC, États-Unis

Schukken $\mathrm{YH}$, Buurman J, Huirne RBM, Willemse $A H$, Vernvoy JCM, van der Broek J, Verheijden JHM (1994) Evaluation of optimal age at first conception in gilts from data collected in commercial swine herds. $J$ Anim Sci 72, 1387-1392

Tess MW, Bennett GL, Dickerson GE (1983) Simulation of genetic changes in life cycle efficiency of pork production. I. A bioeconomic model. J Anim Sci 56, 336-353

Vermeer HM, Slijkhuis A (1989) Insemination breeding sows at the first or second oestrus. Proefverslag Proefstation voor de Varkenshouderij, $20 \mathrm{p}$

Young LG, King GJ, Walton JS, MacMillan I, Klevorick M (1990) Reproductive performance over four parities of gilts stimulated to early oestrus and mated at first, second or third observed oestrus. Can J Anim Sci70, 483-492 